\title{
Influence of two guidance techniques on verbal learning ${ }^{1}$
}

\author{
JOSEPH B. SIDOWSKI, SAN DIEGO STATE COLLEGE \\ MARK GREENE, UNIVERSITY OF OREGON ${ }^{2}$
}

Subjects in two experiments learned lists of paired associates with or without prompting or first letter cues, Recall scores on interspersed test trials, and seven days later, indicated an expected superiority for prompting but no significant cue effects.

The experiments reported here deal with the effects of two types of guidance on paired associate learning. The first, prompting, refers to a set of operations in which the learner makes no incorrect response during training. The correct $S-R$ connection is given on each training trial, and $S$ merely repeats the response term just presented. Retention is measured during one or more test trials in which only stimuli are presented and $S$ required to recall the correct response with neither stimulus support nor feedback. Although a relatively "easy" way to learn, prompting has proven superior to confirmation in a number of studies (Cook \& Kendler, 1956; Cook, 1958; Cook \& Spitzer, 1960; Reynolds, 1965, 1967; Sidowski, Kopstein, \& Shillestad, 1961). Methodologically, this advantage has been cited as being due to (1) Ss committing no errors during training, and (2) S-R terms being connected closely or concurrently in time (Cook \& Kendler, 1956). Anticipation methods, on the other hand, allow $S$ to connect the wrong response to the stimulus, if he is incorrect, and provide a time gap between the presentation of the stimulus and correct knowledge of results. Unfortunately, prompting also allows more practice time, a fact left unmentioned in numerous studies to date.

Our second guidance technique, cueing, offers stimulus support by providing the first letter of the item to be learned during the response interval of each training trial. Obviously, if there is an advantage to this type of guidance, it should be most noticeable for the confirmation method where $S$ is required to sustain a memory search on each trial before committing a response. However, it is possible that although storage is enhanced, retrieval is not because of the difference in stimulus conditions between training (cue present) and test (cue absent).

Method

In Experiment I Ss were instructed to learn a list of English words and their Japanese equivalents (Hepburn spelling ${ }^{3}$ ). All words consisted of four letters, but no term in either language list began with the same letter or ended with the same syllable. In addition, neither the English nor Japanese word of any pair began or ended with the same letters.
The Ss were subjected to nine training trials interspersed with recall tests on Trials 4, 8, and 12. Only the English word was shown on test trials and $S$ required to write the Japanese equivalent with no feedback.

For the confirmation conditions, Ss saw the stimulus term (English), anticipated the response (Japanese), then received knowledge of results. Prompting groups saw the stimulus, then the response, and immediately wrote the response term just presented. Cue subgroups were shown the first letter of the Japanese term during the response interval.

Each word of the paired associate was individually flashed onto a screen at a 2 sec rate by a Dunning Animatic filmstrip projector. Ss were allowed $6 \mathrm{sec}$ to respond; the inter-pair interval was $1 \mathrm{sec}$. On test trials, the stimulus was flashed for $2 \mathrm{sec}$, and $6 \mathrm{sec}$ allowed for recall. Different random orders of the list were given on each training and test trial.

Seven days later, Ss were returned for an additional test. Only English words were presented, one at a time, and $20 \mathrm{sec}$ allowed for each response.

Experiment II replicated Experiment $I$ in all respects except for list content; the paired associates consisted of digit-nonsense syllable combinations. Single digits 0-9 were each assigned at random to one of the nonsense syllables. The latter were CVC trigrams of $25 \%$ association value (Archer, 1960). No two syllables began or ended in the same consonant.

Sixty different Ss with no formal training in Oriental languages and no experience in verbal learning research served in each experiment. They were students from introductory psychology courses at San Diego State College who received class credit. All were run individually, and assignment of Ss to conditions was random. Results

Analyses of variance on total number of correct responses in both experiments indicated a significant superiority for prompting on each of the three initial test trials $(p<.01)$ and seven days later $(p<.05)$.

These effects were due primarily to the larger number of extra-list intrusions $(p<.01)$ for the confirmation condition; intra-list intrusions contributed no significant effect in either study.

There were no significant interaction effects, no difference in the number of first letters correctly recalled, and test trial performance for Ss trained with first letter cues was not significantly better than non-cue groups in either experiment. In fact, the mean numbers 
of correct responses were consistently larger for the latter, with or without prompting. These results agree with data presented earlier (Greene \& Sidowski, 1963). Discussion

Of course it is tempting to evaluate the superiority of prompting within the framework of a mediation theory proposed by Cook \& Kendler (1956) and propounded by Aiken \& Lau (1967), but justifying such an interpretation would be most difficult. The prompting method, as operationally defined by Cook \& Kendler and subsequently by most other researchers in the area, simply allows more time for rehearsal if ss care to use it. Results of several studies in this laboratory suggest that they do.

Apparently the lack of first letter cues during recall hinders retrieval for Ss trained with cues. But this tells us little about their effects on storage, so the training trial data were evaluated for the confirmation conditions (obviously, prompting groups showed no error). Analysis showed the confirmation-cue group producing fewer errors than non-cue during the two three-trial blocks preceding Tests 1 and 2 but not Block 3. So storage was enhanced, at least over the early trials.

Explaining this result involves numerous problems of memory search and coding, only a few of which are noted here. There was a discrimination difference between training (cue) and test (non-cue) that complicated search; the first letter of the four or three letter chain was no longer presented as an eliciting stimulus. But after the initial test trials, Ss should have learned this fact and adjusted accordingly. Whether this occurred is difficult to determine from the data, although analyses showed no differential effects of cue vs non-cue over test trials. A more objective but tangential explanation is offered by the lack of significant difference between cue and non-cue groups in number of first letters correctly recalled during tests. Apparently the first letter cue was encoded as one unit with little overt repetition. The remaining letters of an item were encoded and stored as a separate more actively emphasized chunk. Examination of the data and post-experimental questioning strongly support this assumption. But other explanations are obvious as well as conjectural. Further clarification requires research utilizing cues in training, test, and both.

References

AIKEN, E. G., \& LAU, A. W. Response prompting and response confirmation: A review of recent literature. Psychol. Bull., 1967, 5, 330-341.

ARCHER, E. J. A re-evaluation of the meaningfulness of all possible CVC trigrams. Psychol. Monogr., 1960, 74, (10, Whole No. 497), $1-23$.

COOK, J. O. Supplementary report: Processes underlying learning a single paired-associate item. $J$. exp. Psychol., 1958, 56, 455.

COOK, J. O., \& KENDLER, T. S. A theoretical model to explain some paired-associate learning data. In G. Finch \& F. Cameron (Eds.) Symposium on Air Force human engineering, personnel, and training research. Wash., D. C.: Nat. Acad. Sci.- Nat. Res. Coun., 1956, 90-98.

COOK, J. O., \& SPITZER, M. E. Supplementary report: Prompting versus confirmation in paired-associate learning. $J$. exp. Psychol, 1960, 59, 275-276.

GREENE, M., \& SIDOWSKI, J. B. Effects of prompting and cueing upon short- and long-term retention of paired-associates. Paper presented at meetings of Western Psychol. Assoc., Los Angeles, 1963.

REYNOLDS, J. H. Paired-associate learning by anticipation and nonanticipation methods: Supplementary data. Psychol. Rep., 1965, $16,1123-1124$.

REYNOLDS, J. H. Confirmation, contiguity, and response practice in paired-associate learning. J. exp. Psychol, 1967, 73, $394-400$.

SIDOWSKI, J. B., KOPSTEIN, F. F., \& SHILLESTAD, I. J. Prompting and confirmation variables in verbal learning. Psychol. Rep., 1961, $8,401-406$.

Note

1. This research was supported, in part, by Grant 7-1-104, U. S. Office of Education.

2. Now at American Institute of Research, Palo Alto.

3. Japanese phrase book. TM 30-641. Wash., D. C.: U. S. War Dept., 1942. 